\title{
Optical modeling of Fresnel zoneplate microscopes
}

\author{
Patrick P. Naulleau, Iacopo Mochi, and Kenneth A. Goldberg
}

Center for X-Ray Optics, Lawrence Berkeley National Laboratory, Berkeley, CA 94720

\begin{abstract}
Defect free masks remain as one of the most significant challenges facing the commercialization of extreme ultraviolet (EUV) lithography. Progress on this front requires high performance wavelengthspecific metrology of EUV masks including high resolution and aerial-image microscopy performed near 13.5-nm wavelength. Arguably the most cost effective and rapid path to proliferating this capability is through the development of Fresnel zoneplate based microscopes. Given the relative obscurity of such systems, however, modeling tools are not necessarily optimized to deal with them and their imaging properties are poorly understood.

Here we present a modeling methodology to analyze zoneplate microscopes based on commercially available optical modeling software and use the technique to investigate the imaging performance of an off-axis EUV microscope design. The modeling predicts that superior performance can be achieved by tilting the zoneplate, making it perpendicular to the chief ray at the center of the field, while designing the zoneplate to explicitly work in that tilted plane.

Although the examples presented here are in the realm of EUV mask inspection, the methods described and analysis results are broadly applicable to zoneplate microscopes in general including full field soft-x-ray microscopes routinely used in the synchrotron community.
\end{abstract}

Keyword: extreme ultraviolet, lithography, Fresnel zoneplate, aberrations, system analysis 


\section{Introduction}

Defect free masks remain as one of the most significant challenges facing the commercialization of extreme ultraviolet (EUV) lithography. Given the wavelength-specific response of multilayer-coated and patterned reflective masks, progress in mask defect detection, mitigation, and repair all require high performance at-wavelength metrology of EUV masks and in particular at-wavelength aerialimage microscopy.

Arguably the most cost effective and rapid path to proliferating this capability is through the development of Fresnel zoneplate based microscopes. The reason for this is that the system is relatively simple, with the imaging path being comprised of a single, diffractive, high-magnification objective lens that is sub-mm in dimension. Successful examples of such tools are the SEMATECH Berkeley Actinic Mask Inspection Tool (AIT) which has proven the utility of such a concept [1], and the EUV microscope developed by Colorado State University, which has demonstrated a stand-alone prototype [2]. Given the relative obscurity of such systems, however, their imaging properties are arguably poorly understood.

Here we present a modeling methodology to analyze zoneplate microscopes based on commercially available optical modeling software, and use the technique to investigate the imaging performance of an off-axis EUV microscope design nominally matching that of the AIT. This analysis method has recently been used to develop an optimized alignment procedure for the AIT [3]. Although the motivation presented here is in the realm of EUV mask inspection, the methods described and analysis results are broadly applicable to zoneplate microscopes in general including full field soft-x-ray microscopes routinely used in the synchrotron community [4-6].

\section{System description}

A typical system configuration for an EUV mask microscope is shown in Fig. 1. Note that for 
clarity, the distances and angles are not to scale, and only the chief illumination ray is shown. In practice, the chief illumination ray angle of incidence (AOI) is set to the same AOI as used in the lithography tool which is currently $6^{\circ}$. The zoneplate is mounted parallel to the mask, meaning that the chief ray also strikes the zoneplate at an AOI of $6^{\circ}$. Furthermore, the zoneplate is designed to work off axis such that the first-order imaging beam is bent back by $6^{\circ}$ to exit the zoneplate perpendicular to its surface and that of the mask. Thus the chief ray leaves the zoneplate perpendicular to the surface [1]. The off-axis design is achieved by placing the zoneplate, which can be described as a hologram of a point source onto a spatial carrier (i.e. an off-axis hologram). An equivalent description is that the off-axis zoneplate is a displaced subaperture of a larger, on-axis parent zoneplate.

The benefit of the off-axis configuration is that it separates the zeroth order from the first diffracted order at the CCD without the use of an order sorting aperture. This simplifies the design and optimizes imaging contrast. A drawback of the off-axis configuration, however, is that the dispersion is increased and a narrower bandwidth illumination must be used to avoid chromatic blur. The increase in dispersion comes from the fact that the nominal pitch of the zoneplate is decreased relative to an equivalent NA on-axis zoneplate. While this is of little concern for synchrotron implementations, it poses significant challenges for application of the technique using stand-alone sources [7-9].

As with any optical system, the magnification is the ratio of the image-plane distance to object-plane distance. In practice, EUV mask microscope systems strive for magnification ratios on the order of 1000. For a typical AIT configuration, this translates to a zoneplate distance of approximately $1 \mathrm{~mm}$ and a CCD distance of approximately $680 \mathrm{~mm}$.

\section{Modeling methodology}


The optical system modeling method presented here is based on ZEMAX [10], however, the method should be readily applicable using other optical modeling packages. The system is modeled in reverse with the object plane in the model being the CCD and the image plane being the mask. All subsequent references to these terms will follow this convention. Following the AIT configuration, and the model convention, the object-side chief ray is perpendicular to the zoneplate. The distance from the object plane to the zoneplate is $680 \mathrm{~mm}$ and the distance from the zoneplate plane to the image plane is approximately $1 \mathrm{~mm}$. Since the AIT is designed to emulate lithography tools with $4 \times$ demagnification and NA values of 0.25 and higher, we use a mask-side (image-plane) NA value of 0.0625 . The zoneplate lens is modeled as an ideal thin phase plane with the phase being that of an off-axis sphere. The sphere radius is set to $1 \mathrm{~mm}$ and the pupil offset is $105 \mu \mathrm{m}$. The offset is determined based on the target angle at the mask $\left(6^{\circ}\right)$ and the working distance. In defining the zoneplate this way, we accurately model the wavefront in the first diffracted order, including the spatial carrier but neglect the other diffraction orders. If on the other hand we were to model a system where background order contamination is important, we could account for the contamination as flare with little loss of generality.

\section{Field-dependent aberrations}

Figure 2 shows a portion of the optical model, zoomed in to the zoneplate and image plane (mask) region. Three field points are included: one on axis and the other two at $\pm 4 \mu \mathrm{m}$ in the $y$ direction at the image plane, respectively. The expected $6^{\circ}$ deflection and focusing of the rays is observed. Zooming in even more tightly to the image plane region [Fig. 3(a)], it is evident that the off-axis zoneplate suffers from a tilted image plane, creating a focus shift across the field. A similar view can be obtained for the $x$-axis of the image plane [Fig. 3(b)] where we see no evidence of field tilt. 
To get a better idea of the imaging performance we next consider through-focus spot diagrams for five field points of interest (Fig. 4). The field positions called out in Fig. 4 are in object space and thus 680 times larger than the field position mentioned above. As indicated by the tilted field in Fig. 2, analysis of the circle of least confusion shows approximately 1- $\mu \mathrm{m}$ of focus shift at the off-axis positions in the $y$ direction (at $\pm 4 \mu \mathrm{m}$ ). We also see evidence of significant levels of astigmatism as we move off axis. For $y$ displacements, we see $0^{\circ}$ astigmatism, and for $x$ displacements where there is no field tilt, we observe $45^{\circ}$ astigmatism of comparable magnitude to the $0^{\circ}$ astigmatism observed with $y$ field displacement.

More careful analysis of optimal circle of least confusion performance reveals a field tilt of $12^{\circ}$ in the $y$ direction. This value is significantly higher than one might expect based on the geometrical change in effective image distance as we move off axis. Starting with a nominal field offset of $105 \mu \mathrm{m}$ and nominal image to zoneplate plane separation of $1 \mathrm{~mm}$ it is simple to show that changing the field offset to $100 \mu \mathrm{m}$, for example, would change the effective image distance from $\operatorname{sqrt}\left(1000^{\wedge} 2+105^{\wedge} 2\right)$ to $\operatorname{sqrt}\left(1000^{\wedge} 2+100^{\wedge} 2\right)$ or $1005.5 \mu \mathrm{m}$ to $1005.0 \mu \mathrm{m}$. This $0.5-\mu \mathrm{m}$ effective image shift over a $5-\mu \mathrm{m}$ separation yields only $5.7^{\circ}$ of field tilt, which corresponds approximately to the AOI on the mask but is off by more than a factor of two compared to the true image plane tilt as determined by the model.

Next we consider the actual aberration magnitudes across the field. The resulting Fringe Zernike magnitudes in waves are shown in Table 1. As expected from the results above, the primary field-dependent aberrations are defocus and astigmatism. The maximum individual Fringe Zernike magnitudes are approximately $\lambda / 20$. Coma and spherical error remain well below $\lambda / 75$ across the field of view. 
The above results assume an effective $4 \times \mathrm{NA}$ of 0.25 . The impact of increasing NA on aberrations is illustrated in Fig. 5 which shows a plot of the astigmatism and coma magnitude as a function of effective $\mathrm{NA}$ at image plane field location $(0,4 \mu \mathrm{m})$. It is evident that the increasing aberrations at the off-axis field point lead to a reduced field size. This effect is quantified in Fig. 6 which shows the equivalent astigmatism magnitude field radius as a function of effective NA. Astigmatism was chosen because it is the dominant aberration. We note, however, that the dominance of the astigmatism does decrease as the NA is increased because coma increases faster as a function of NA than does astigmatism (Fig. 6). Nevertheless, even at an effective $4 \times \mathrm{NA}$ of 0.75 , the astigmatism magnitude at the edge of the field is nearly twice as large as the coma. Increasing the effective NA from 0.25 to 0.75 yields an equivalent aberration field width reduction of nearly $10 \times$.

\section{Effect of focal length}

The results above show that for an astigmatism limit of approximately $\lambda / 20$, the maximum field radius is limited to approximately $4 \mu \mathrm{m}$. Given a zoneplate radius of $62.5 \mu \mathrm{m}$, this corresponds to a relative field radius of $6.4 \%$. This metric raises the question of the impact of focal length, for example, does the absolute field size shrink as the focal length and, thus, zoneplate radius are decreased? Table 2 shows the Fringe Zernike magnitudes in waves for essentially the same system studied above, but with the focal length reduced to $0.5 \mathrm{~mm}$. The NA, AOI, and object distance are kept fixed. Given the fixed object distance, the magnification is increased to 1360. The aberrations are reported at the off-axis field point of $(0.4 \mu \mathrm{m}, 0.4 \mu \mathrm{m})$ in the image plane, which corresponds to $(5.44 \mathrm{~mm}, 5.44 \mathrm{~mm})$ in the object plane. Table 2 shows that the absolute field size is nearly independent of focal length; thus, for the $0.5-\mathrm{mm}$ focal length case, the relative field size increases to $12.8 \%$. Repeating these simulations with the object distance 
decreased to $340 \mathrm{~mm}$, thereby bring the magnification back to 680, yields essentially identical results showing that the results are also independent of magnification.

\section{Minimizing field tilt}

Finally we ask the question of whether or not we can minimize the field tilt. The obvious solution would be to tilt the image plane, yet the image plane in our model is the surface of the mask sample we wish to study, and we require an AOI of $6^{\circ}$ on the mask to emulate the illumination of a mask in a stepper, thus we cannot implement any correction there. Given the extremely large magnification of the system, it is also not possible to implement a correction by tilting the CCD (object plane in our model). This leaves only the zoneplate itself; however, with the zoneplate being in a conjugate plane, tilting it would not have the desired effect. Nevertheless, we still consider the case of the zoneplate tilted to be perpendicular to the imageside chief ray. Figure 7 shows the resulting through-focus spot diagrams ( $2 \mu \mathrm{m}$ focus steps) as a function of field position, and Table 3 lists the computed aberrations. Although the field tilt has been mitigated (reduced by a factor of two based on the Zernike defocus term), wavefront performance suffers significantly with very large astigmatism and coma values: this is also evident in the spot diagrams. Given that the zoneplate was designed based on an un-tilted geometry, these results should come as no surprise.

Extending the Fourier transform holography analogy discussed above, it can be shown that modification of the field tilt could also be achieved with a lateral offset in the zoneplate plane (Fourier transform theory tells us that a lateral shift in one domain yields linear phase in the conjugate domain). In this system, however, the zoneplate offset is not a free parameter since it is determined by the required AOI on the mask. Thus zoneplate offset cannot be used to reduce the tilt error. 
Finally we reconsider the case where the zoneplate is tilted perpendicular to the imageside chief ray, but this time the zoneplate is re-designed explicitly to work in a tilted plane. This is achieved by computing the point source hologram over the desired tilted plane which is set to match the AOI on the mask. Figure 8 shows the spot diagram results and Table 4 the aberrations. This configuration produces a dramatic reduction in astigmatism, greater than a factor of 10 , and a factor of two reduction of the field tilt. Little change in coma and spherical aberration magnitude is observed.

\section{Summary}

A modeling methodology for zoneplate microscopes has been presented and used to analyze the theoretical performance of an EUV mask inspection microscope. The results show that superior performance can be achieved by re-designing the zoneplate to work in a tilted plane, perpendicular to the chief ray at the center of the field. Doing so yields a greater than $10 \times$ reduction in astigmatism error across the field and $2 \times$ reduction in field tilt with little impact on coma and spherical aberration.

This work was performed at Lawrence Berkeley National Laboratory and supported by SEMATECH through the U.S. Department of Energy under Contract No. DE-AC02$05 \mathrm{CH} 11231$.

\section{References}

1. K. A. Goldberg, P. Naulleau, I. Mochi, E. H. Anderson, S. B. Rekawa, C. D. Kemp, and R. F. Gunion, H.-S. Han, S. Huh, “Actinic extreme ultraviolet mask inspection beyond 0.25 numerical aperture," Journal of Vacuum Science and Technology B 26 (6), 2220-4 (2008).

2. F. Brizuela, S. Carbajo, A. Sakdinawat, D. Alessi, D. H. Martz, Y. Wang, B. Luther, K. A. Goldberg, I. Mochi, D. T. Attwood, B. La Fontaine, J. J. Rocca, and C. S. Menoni, "Extreme 
ultraviolet laser-based table-top aerial image metrology of lithographic masks,” Opt. Exp. 18 (14), 14467-73 (2010).

3. I. Mochi, K. A. Goldberg, P. Naulleau, S. Huh, "Improving the performance of the Actinic Inspection Tool with an optimized alignment procedure," Proc. SPIE 7271,76361A-1-9 (2009).

4. P. Fischer, M.-Y. Im, "Imaging nanoscale magnetic structures with polarized soft X-ray photons," IEEE Photonics Journal 2, 253 (2010).

5. W. Chao, J. Kim, S. Rekawa, P. Fischer, E.H. Anderson, "Demonstration of $12 \mathrm{~nm}$ Resolution Fresnel Zone Plate Lens based Soft X-ray Microscopy,” Optics Letters 17, 17669 (2009).

6. D. Attwood, W. Chao, E. Anderson, J. A. Liddle, B. Harteneck, P. Fischer, G. Schneider, M. Le Gros and C. Larabell, "Imaging at High Spatial Resolution: Soft X-Ray Microscopy to 15 nm” Journal of Biomedical Nanotechnology 2, 75 (2006).

7. I. McKinnie, H. Kapteyn, "High-harmonic generation: Ultrafast lasers yield X-rays," Nature Photonics 4, 149 (2010).

8. P. Blackborow, M. Partlow, S. Horne, M. Besen, D. Smith, D. Gustafson, "EUV source development for AIMS and blank inspection," Proc. SPIE 7636, 763609 (2010).

9. N. Farrar, D. Brandt, I. Fomenkov, A. Ershov, N. Bowering, W. Partlo, D. Myers, A. Bykanov, G. Vaschenko, O. Khodykin, J. Hoffman, C. Chrobak, "EUV laser produced plasma source development," Microelectronic Engineering 86, 509 (2009).

10. ZEMAX optical system design software is developed and distributed be ZEMAX Development Corporation, 3001 112th Avenue NE, Suite 202, Bellevue, WA 98004-8017 USA.

\section{List of Figures}


Fig. 1. Schematic of typical system configuration for an EUV mask microscope. Distances and angles are not to scale. Only the chief illumination ray is shown.

Fig. 2. Zoneplate/image plane (mask) region of the optical model showing 3 field points: one on axis and the other two at $+/-4 \mu \mathrm{m}$ in $y$ at the image plane, respectively

Fig. 3. A $y-z$ plane view of image region (a) showing that the off-axis zoneplate suffers from a tilted image plane in the $y$ direction. A $x z$-plane view of image region (b) showing no evidence of field tilt along the $x$-axis.

Fig. 4. Through-focus spot diagrams for 5 field points. The field positions are in object space and thus 680 times larger than the corresponding image (mask) plane offsets.

Fig. 5. Plot of astigmatism and coma magnitude in waves at image plane field point $(0,4 \mu \mathrm{m})$ as function of effective NA.

Fig. 6. Plot of equivalent astigmatism magnitude field radius as function of effective NA.

Fig. 7. Cross-field through focus spot diagrams for system with zoneplate tilted to be perpendicular to the central field point chief ray.

Fig. 8. Cross-field through focus spot diagrams for system with zoneplate tilted to be perpendicular to the central field point chief ray and explicitly designed to work in titled plane.

\section{List of Tables}

Table 1. Cross-field aberrations for baseline configuration. Corresponding spot diagrams are shown in Fig. 4.

Table 2. Zernike aberrations at off-axis image-plane field point $(0 \mu \mathrm{m}, 4 \mu \mathrm{m})$ for two different focal length zoneplate systems with the same NA, AOI, and object distance. Note that nearly identical results are obtained when the magnification instead of the object distance is kept fixed. 
Table 3. Cross-field aberrations for baseline zoneplate titled to be perpendicular to the central field point chief ray. Corresponding spot diagrams are shown in Fig. 7.

Table 4. Cross-field aberrations for a baseline zoneplate, titled into the plane perpendicular to the central field point chief ray and designed to work in the titled plane. Corresponding spot diagrams are shown in Fig. 8. 


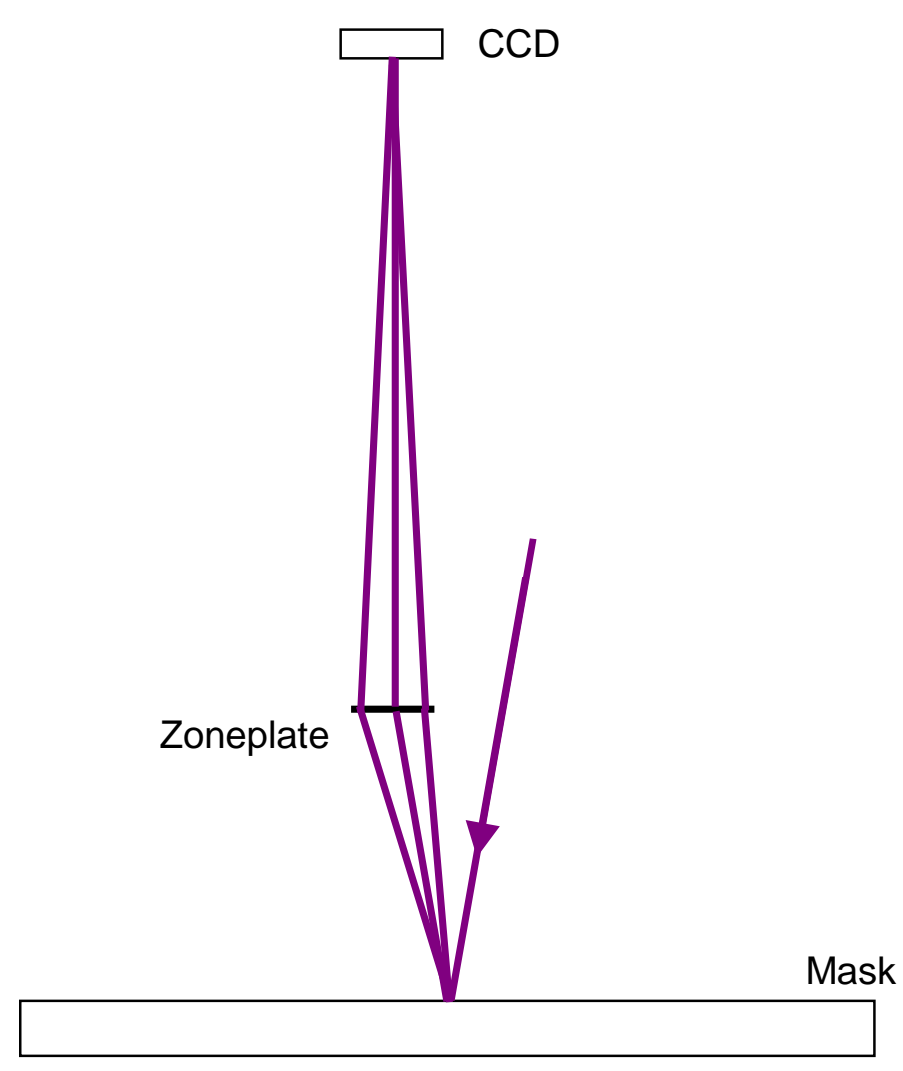

Fig. 1. Schematic of typical system configuration for an EUV mask microscope. Distances and angles are not to scale. Only the chief illumination ray is shown. 


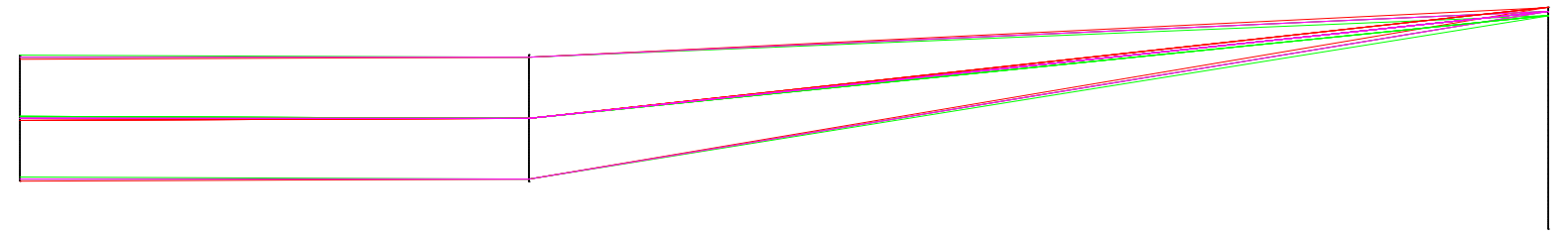

Fig. 2. Zoneplate/image plane (mask) region of the optical model showing 3 field points: one on axis and the other two at $+/-4 \mu \mathrm{m}$ in $y$ at the image plane, respectively 
(a)

(b)

Fig. 3. A $y-z$ plane view of image region (a) showing that the off-axis zoneplate suffers from a tilted image plane in the $y$ direction. A $x z$-plane view of image region (b) showing no evidence of field tilt along the $x$-axis. 


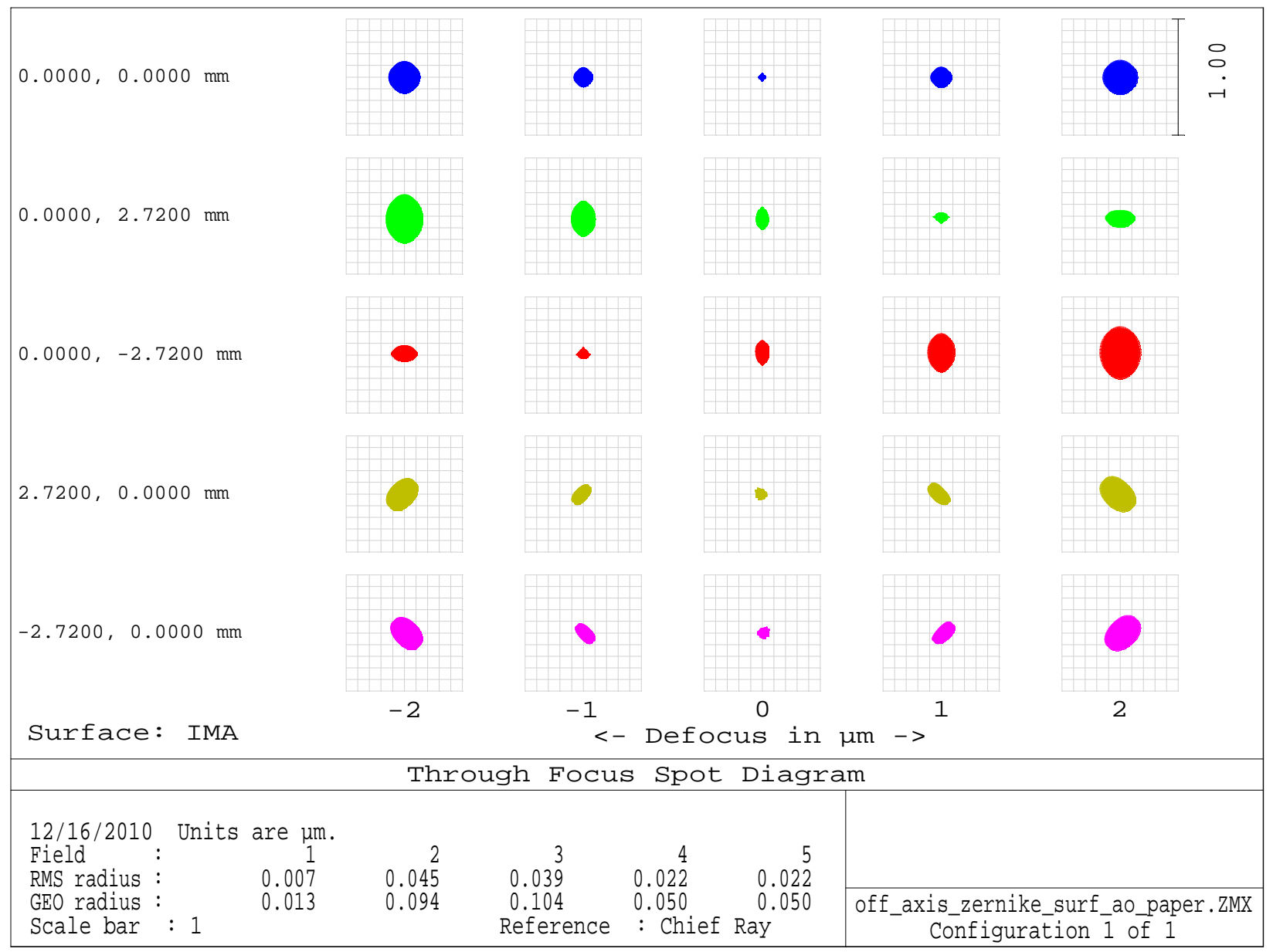

Fig. 4. Through-focus spot diagrams for 5 field points. The field positions are in object space and thus 680 times larger than the corresponding image (mask) plane offsets. 


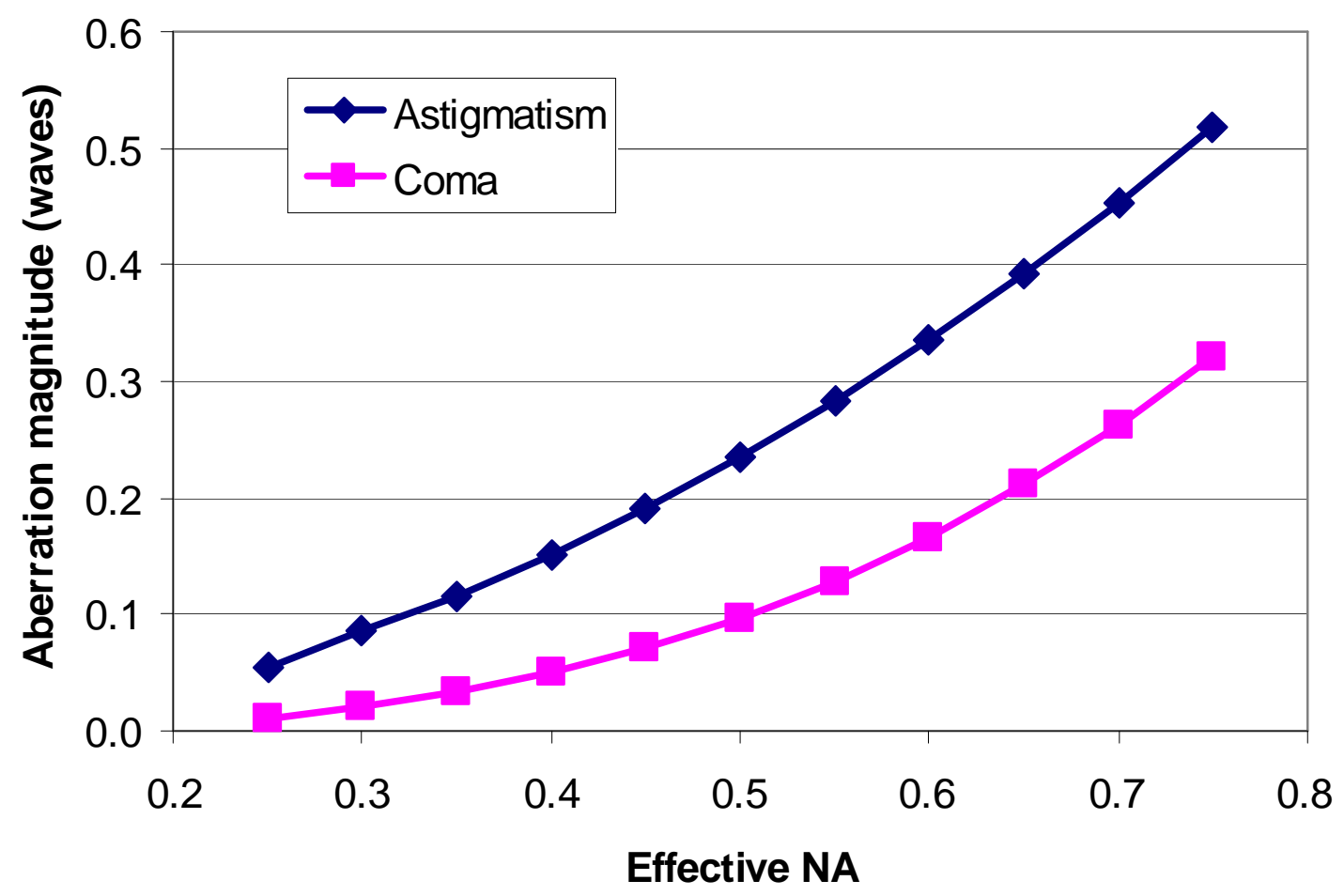

Fig. 5. Plot of astigmatism and coma magnitude in waves at image plane field point $(0,4 \mu \mathrm{m})$ as function of effective NA. 


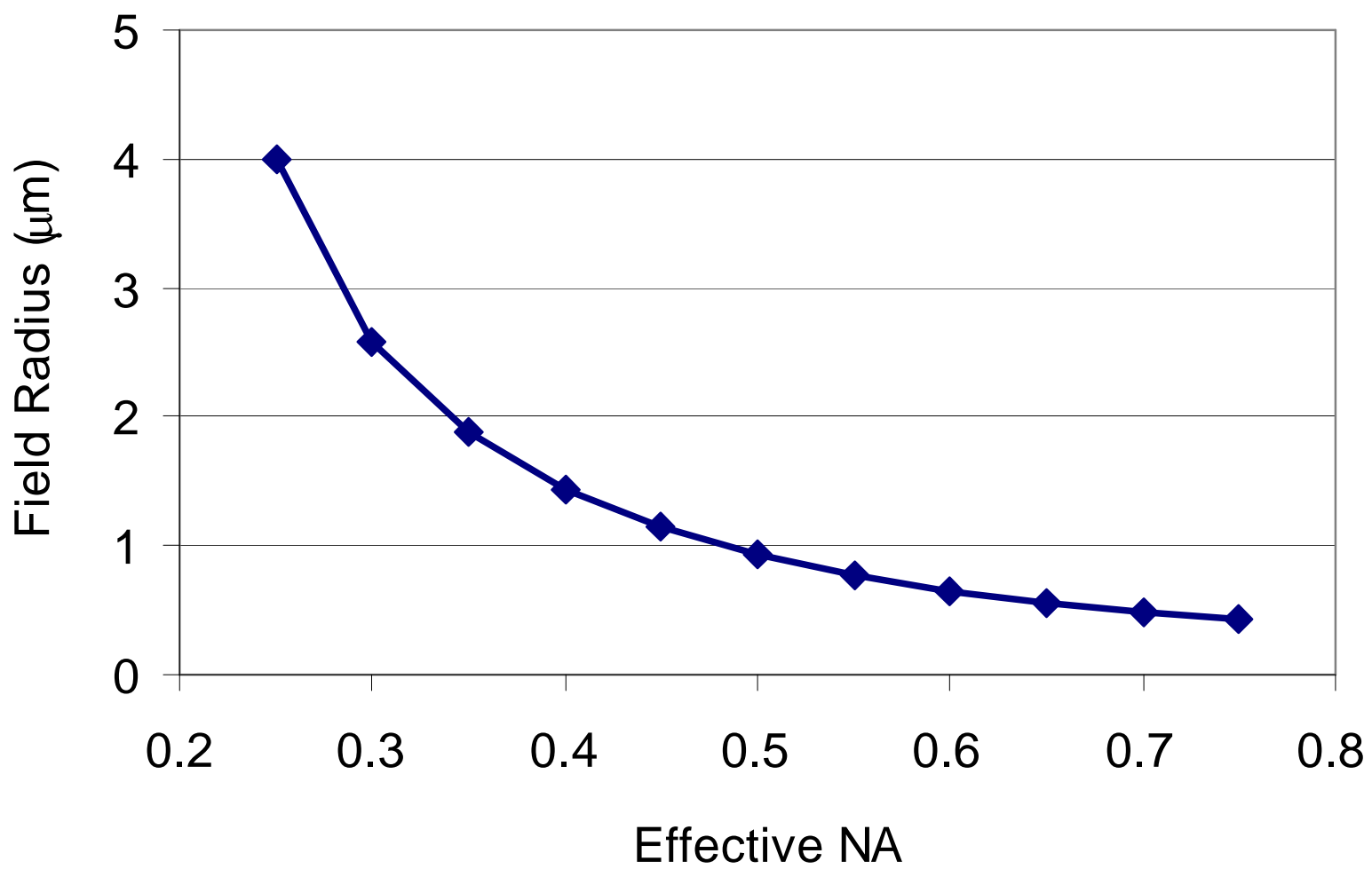

Fig. 6. Plot of equivalent astigmatism magnitude field radius as function of effective NA. 


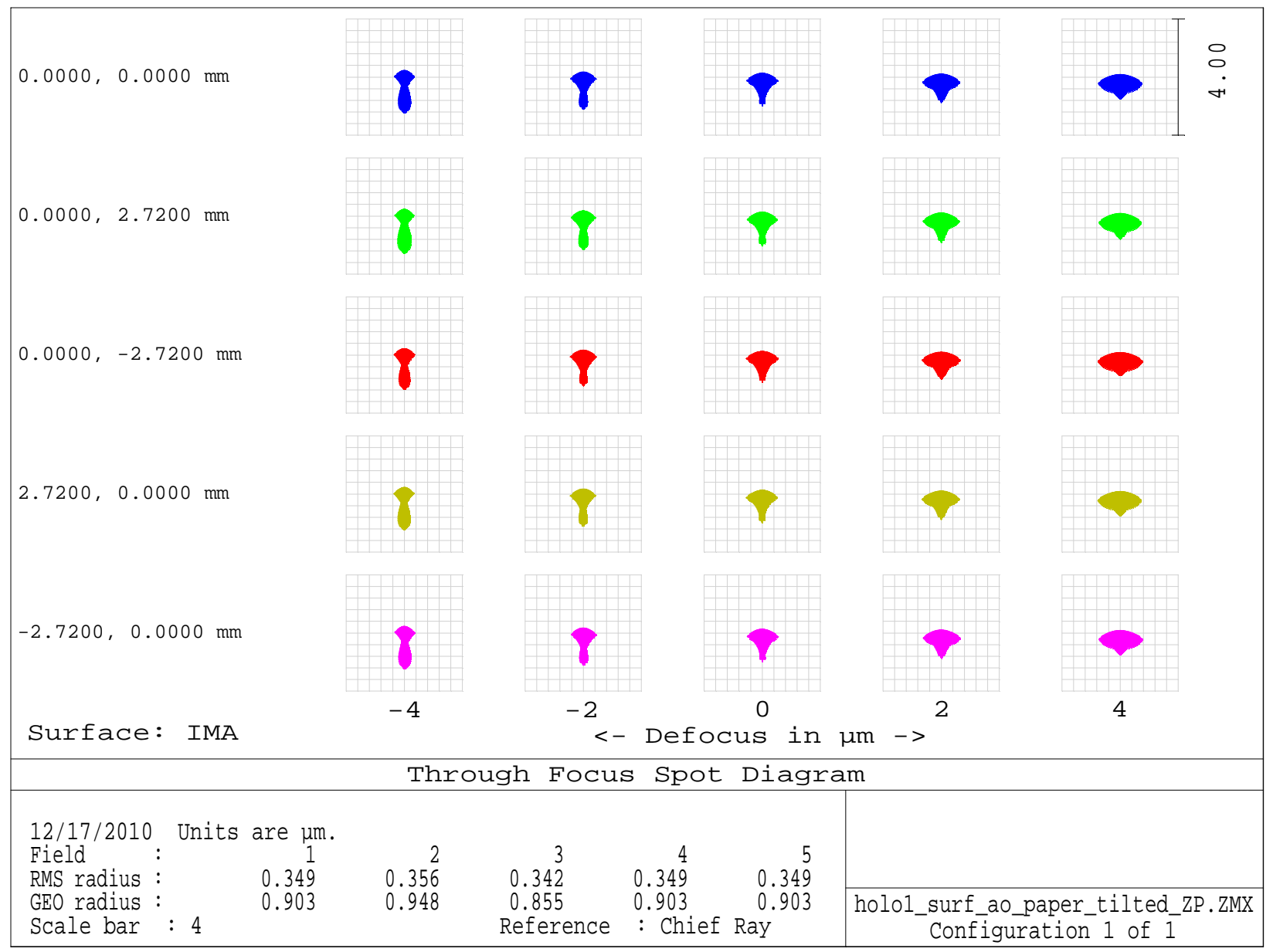

Fig. 6. Cross-field through focus spot diagrams for system with zoneplate tilted to be perpendicular to the central field point chief ray. 


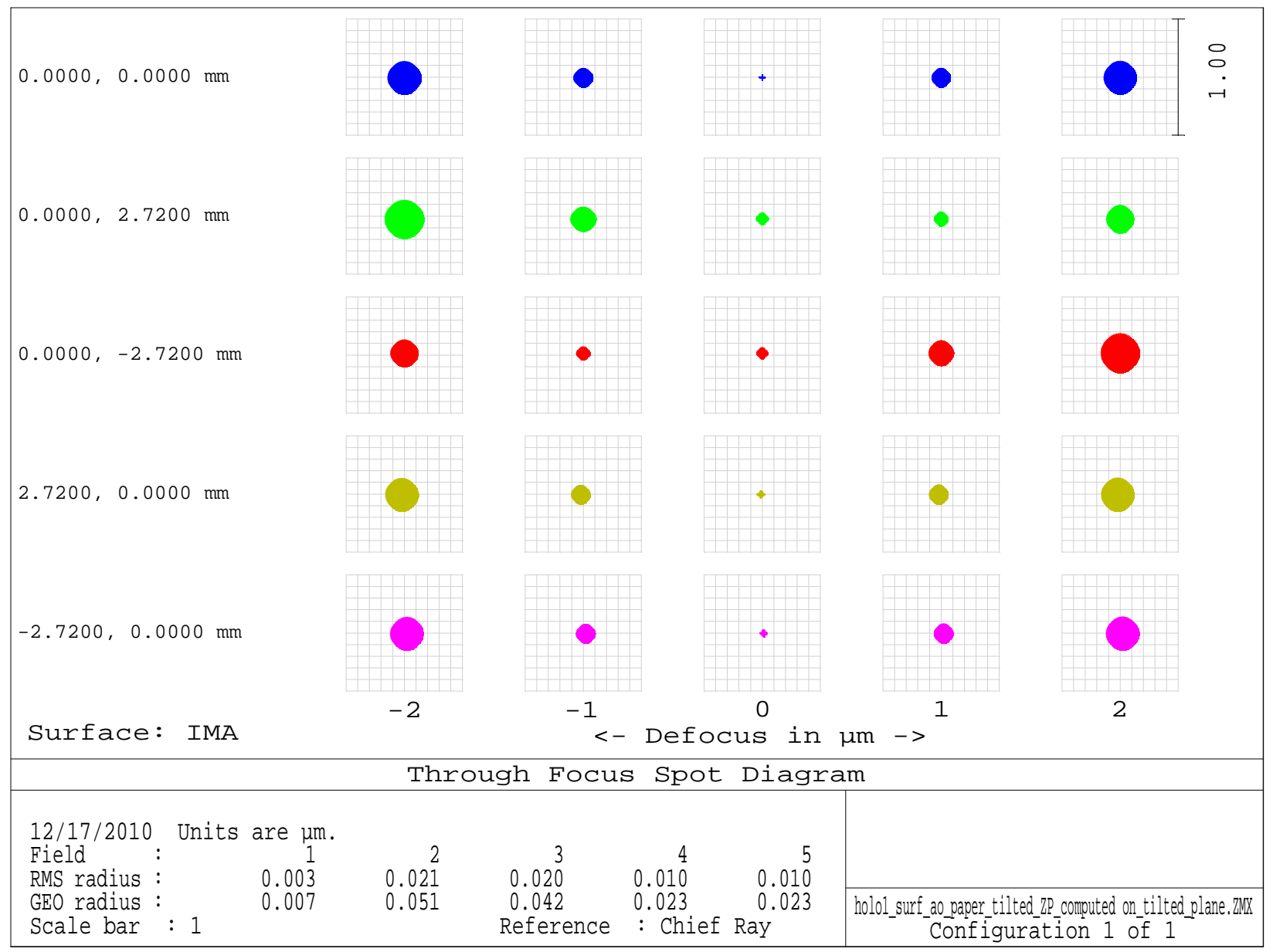

Fig. 7. Cross-field through focus spot diagrams for system with zoneplate tilted to be perpendicular to the central field point chief ray and explicitly designed to work in titled plane. 


\begin{tabular}{|c|c|c|c|c|c|}
\hline \multirow[b]{2}{*}{ Zernike \# } & \multicolumn{5}{|c|}{ Field Point at Mask $(x, y)$} \\
\hline & 0,0 & $0,4 \mu \mathrm{m}$ & $0,-4 \mu \mathrm{m}$ & $4 \mu \mathrm{m}, 0$ & $-4 \mu \mathrm{m}, 0$ \\
\hline 3 (defocus) & 0.0000 & -0.0542 & 0.0563 & 0.0011 & 0.0011 \\
\hline 4 (0 astig.) & 0.0011 & 0.0548 & -0.0547 & 0.0021 & 0.0021 \\
\hline 5 (45ํ astig.) & 0.0000 & 0.0000 & 0.0000 & -0.0551 & 0.0551 \\
\hline 6 (x coma) & 0.0000 & 0.0000 & 0.0000 & 0.0104 & -0.0104 \\
\hline 7 (y coma) & 0.0008 & 0.0108 & -0.0092 & 0.0008 & 0.0008 \\
\hline 8 (spherical) & -0.0001 & 0.0000 & -0.0002 & -0.0001 & -0.0001 \\
\hline
\end{tabular}

Table 1. Cross-field aberrations for baseline configuration. Corresponding spot diagrams are shown in Fig. 4. 


\begin{tabular}{|c|c|c|}
\hline & \multicolumn{2}{|c|}{ Focal length (mm) } \\
\hline Zernike \# & 1 & 0.5 \\
\hline 3 (defocus) & -0.0531 & -0.0510 \\
\hline 4 ( $0^{\circ}$ astig.) & 0.0559 & 0.0552 \\
\hline 5 (45 astig.) & -0.0530 & -0.0509 \\
\hline $6(x$ coma $)$ & 0.0104 & 0.0104 \\
\hline 7 (y coma) & 0.0108 & 0.0103 \\
\hline 8 (spherical) & 0.0000 & 0.0000 \\
\hline
\end{tabular}

Table 2. Zernike aberrations in waves at off-axis image-plane field point $(0 \mu \mathrm{m}, 4 \mu \mathrm{m})$ for two different focal length zoneplate systems with the same NA, AOI, and object distance. Note that nearly identical results are obtained when the magnification instead of the object distance is kept fixed. 


\begin{tabular}{|c|c|c|c|c|c|}
\hline & \multicolumn{5}{|c|}{ Field Point at Mask $(x, y)$} \\
\hline Zernike \# & 0,0 & $0,4 \mu \mathrm{m}$ & $0,-4 \mu \mathrm{m}$ & $4 \mu \mathrm{m}, 0$ & $-4 \mu \mathrm{m}, 0$ \\
\hline 3 (defocus) & 0.0000 & -0.0266 & 0.0287 & 0.0011 & 0.0011 \\
\hline $4\left(0^{\circ}\right.$ astig. $)$ & 0.7230 & 0.7225 & 0.7215 & 0.7241 & 0.7241 \\
\hline 5 (45 astig.) & 0.0000 & 0.0000 & 0.0000 & 0.0004 & -0.0004 \\
\hline $6(x$ coma $)$ & 0.0000 & 0.0000 & 0.0000 & 0.0105 & -0.0105 \\
\hline 7 (y coma) & 0.2764 & 0.2871 & 0.2656 & 0.2764 & 0.2764 \\
\hline 8 (spherical) & 0.0002 & 0.0003 & 0.0002 & 0.0002 & 0.0002 \\
\hline
\end{tabular}

Table 3. Cross-field aberrations for baseline zoneplate titled into the plane perpendicular to the central field point chief ray. Corresponding spot diagrams are shown in Fig. 6. 


\begin{tabular}{|c|c|c|c|c|c|}
\hline & \multicolumn{5}{|c|}{ Field Point at Mask $(x, y)$} \\
\hline Zernike \# & 0,0 & $0,4 \mu \mathrm{m}$ & $0,-4 \mu \mathrm{m}$ & $4 \mu \mathrm{m}, 0$ & $-4 \mu \mathrm{m}, 0$ \\
\hline 3 (defocus) & 0.0000 & -0.0262 & 0.0283 & 0.0011 & 0.0011 \\
\hline $4\left(0^{\circ}\right.$ astig.) & -0.0022 & -0.0036 & -0.0029 & -0.0012 & -0.0012 \\
\hline 5 (45 astig.) & 0.0000 & 0.0000 & 0.0000 & 0.0007 & -0.0007 \\
\hline $6(x$ coma $)$ & 0.0000 & 0.0000 & 0.0000 & 0.0106 & -0.0106 \\
\hline 7 (y coma) & 0.0030 & 0.0137 & -0.0076 & 0.0030 & 0.0030 \\
\hline 8 (spherical) & -0.0002 & -0.0002 & -0.0002 & -0.0002 & -0.0002 \\
\hline
\end{tabular}

Table 4. Cross-field aberrations for a baseline zoneplate, titled into the plane perpendicular to the central field point chief ray and designed to work in the titled plane. Corresponding spot diagrams are shown in Fig. 7. 


\section{DISCLAIMER}

This document was prepared as an account of work sponsored by the United States Government. While this document is believed to contain correct information, neither the United States Government nor any agency thereof, nor The Regents of the University of California, nor any of their employees, makes any warranty, express or implied, or assumes any legal responsibility for the accuracy, completeness, or usefulness of any information, apparatus, product, or process disclosed, or represents that its use would not infringe privately owned rights. Reference herein to any specific commercial product, process, or service by its trade name, trademark, manufacturer, or otherwise, does not necessarily constitute or imply its endorsement, recommendation, or favoring by the United States Government or any agency thereof, or The Regents of the University of California. The views and opinions of authors expressed herein do not necessarily state or reflect those of the United States Government or any agency thereof or The Regents of the University of California. 